\title{
Relación - Hipofisis - Ovario - Testículo
}

\author{
Doctror Antonio llorós Cuéllar \\ Presidente de la Sociedad Colombiana de Endocrinologia \\ Jefe de Consult de Endocrinología del Hospital de San José. \\ Endocrinológo de UNIDIA
}

La esterilidad endocrina puede dividirse en dos capitulos. Uno que estudia las causas endocrinas que secundariamente repercuten sobre el sistema glandular y el ovario en especial y otro que comprende las causas endocrinas intrínsecas. Vos ocuparemos únicamente del segundo.

Las hormonas especificas de la reproduccion son las hormonas sexuales, producidas por el testiculo y el ovario. Sus secreciones están supeditadas al estimulo de una glándula eferente: la hipofisis.

La fisiologia hormonal está representada por una serie de estimulos y contraestímulos que van de la glandula central a la glándula blaneo y viceversa. Y también por la interrelacion que existe entre las glandulas perifericas relacionán dolas siempre con su motor pituitario, en forma tal, que al fallar una de ellas se rompe la armonia y se presenta un disturbio. Tal el case de la falta ovárica bio legica cuya resultante es el climaterium. hoy (1):

La hipofisis anterior produce 3 homonas gonadotropicas conocidas hast.

La Foliculerestimulante o FSH.

La Lutcinizante LH también llamada hormona estimulante de las células intersticiales o ICSH

Y la Prolactina o Lutcotrofina LTH (2).

El FSH tiene accion gametogénica y proveca la formacion de ovulos y es permatozoides. No es capar de producir estrogenos sino en presencia de Lill en el mache no tiene accion demostrada sobe la secrecion de las células inters ticiales. Si se administran simultaneamente FSH y LH se produce una pronun ciada estimulación ovarico uterina. Los estrogenos, testosterona o polvo de ti roides a dosis altas y sostenidas, son capaces de inhibir la producción de FSH ("). 
La hormona luteinizante o LH interviene en la ovulación y estimula la se. creción de estrina y progesterona pero en presencia de FSH o después de su in. tervención. En el macho estimula la producción de andrógenos por las célulav intersticiales del testículo que a su vez son responsables de los caracteres sexuales secundarios específicos de este sexo.

- RELACION JIPOFISIS-OYARIO-

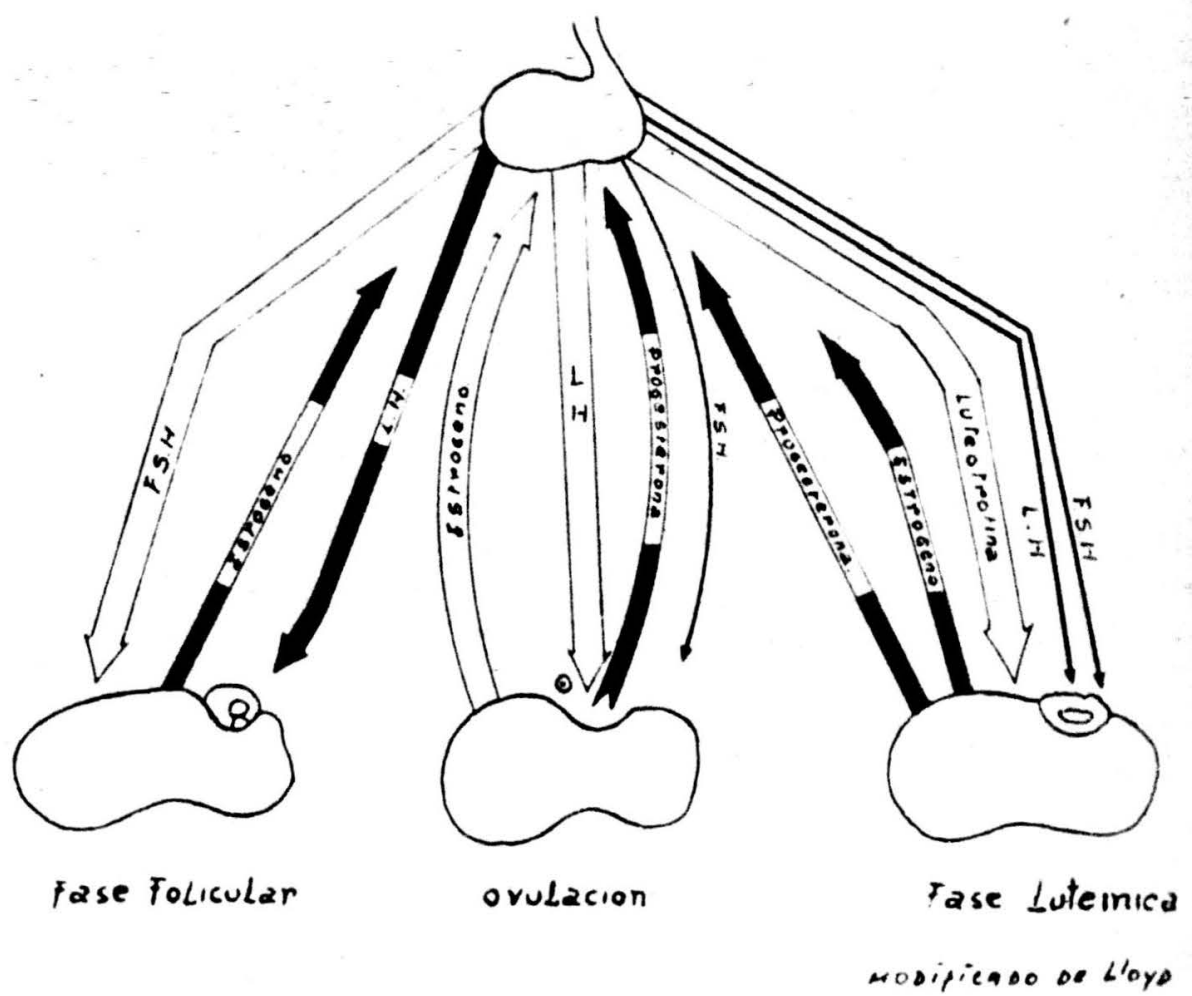

(GRAFICA No 1)

La prolactina o luteotrofina interviene en el mantenimiento de la función secretora en el folículo, cuerpo amarillo y sobre las mamas; no tiene ningún efecto sobre esta última si FSH o LH no han actuado. Naturalmente promucte la lactación fenómeno que es favorecido en el puerperio cuando los niveles de estrógenos y progesterona están bajos y cuando el reflejo de succión favorece 
su produccion. No se ha descrito ningun efecto de esta hormona en el hombr. aunque por su administración se ha obtenido aumento de peso en la préstat. de ratas adultas.

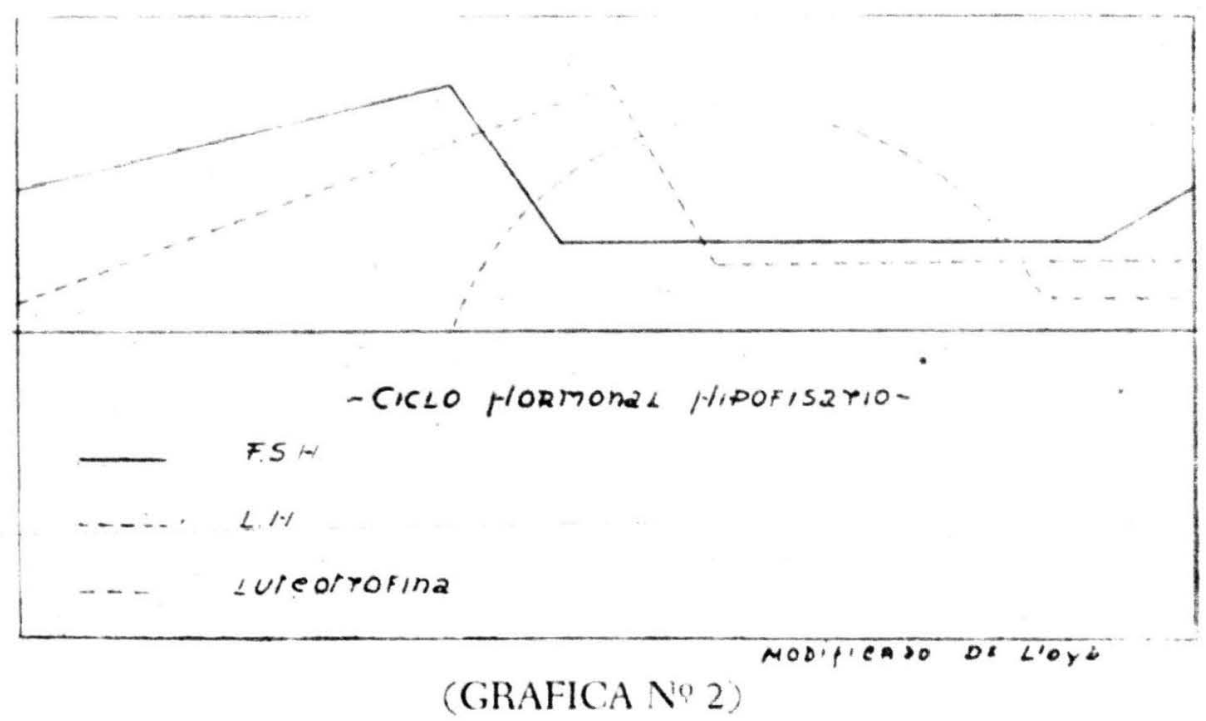

En el cmbaraze hay otra gonadotropina de origen placentario cuya pre sencia aprovecharon Ascheim y Zondek para su prucba. Sc la divide de acuerde con el origen de obtención. Es sérica cuando se usin sueros de yeguas prenadas y coriónica cuande se obtiene de orinas de mujer embarazadas. La primera tiene un efecto gametogenico por su contenide en FSII y la corisnica obraria como IH ( ${ }^{4}$. Vo has que olvidar la forma sincrevica en que obran ni su interrela cion con las hormonas de la glandula blanca.

La funcion de las glandulas sexuales es producir len gametes; ovules y es permatozoides, las hormonas sexuales: estrogenos, progesterona y andrógenos

Estas secreciones se efectuan en distintor grupon celulares.

En el ovario ".) los estrogenos son producidos por los foliculos y el es troma y la progesterona per las células luteinicas del cuerpo amarillo. Hay otras fuenter de preducoien de hormonas sexuales en el onganismo. Los estrógenos so sustancias vecinas del colesterol (") y ácidos biliares cuvos esteres de los de rivados fenólicos on varias veces más activos que la hormona pura. Son inac rivados a parcialmente destruidos tal vee por el higado.

Tiene bajo su dominio directo el desarmllo de los caracteres sexuales pri marios y secundarios átero, ovarios, trompas, vagina, vulva; en la formación de la mama, desarrollo esqueletion ginecoide, en la distribución tipica de la grasa e implantación y caracteres del pelo, en este sentido ha llegado a pro ponerse una prueba del crecimiento del vello axilar como indice cualitativo del 
estado endocrino $\left({ }^{7}\right)$. Tiene acciones definidas en el endometrio y en el epitelio vaginal siendo estas una de las resultantes de su acción tisular. Mantiene niveles sanguíneos típicos durante el ciclo y como ya se dijo, puede inhibir FSH.

La progesterona ovárica aparece únicamente en la segunda mitad del ciclo (A) y ya vimos su relación con las hormonas hipofisarias; es el producto de secre ción del cuerpo amarillo que a su vez ha comenzado a favorecer por el impacto de IH y LTH sobre la cicatriz que ha dejado la postura ovular. Estimula la hipofisis para producir hormon: luteinizante adicional. Produce cambios carat terísticos en el endometrio y en el epitelio vaginal que son aprovechados para evi denciar la ovulación. En el embarazo, se excreta en cantidades progresivamente crecientes. En la orina se encuentra un producto de su metabolismo, el preg nandiol y su estudio es de ayudá en el diagnóstico.

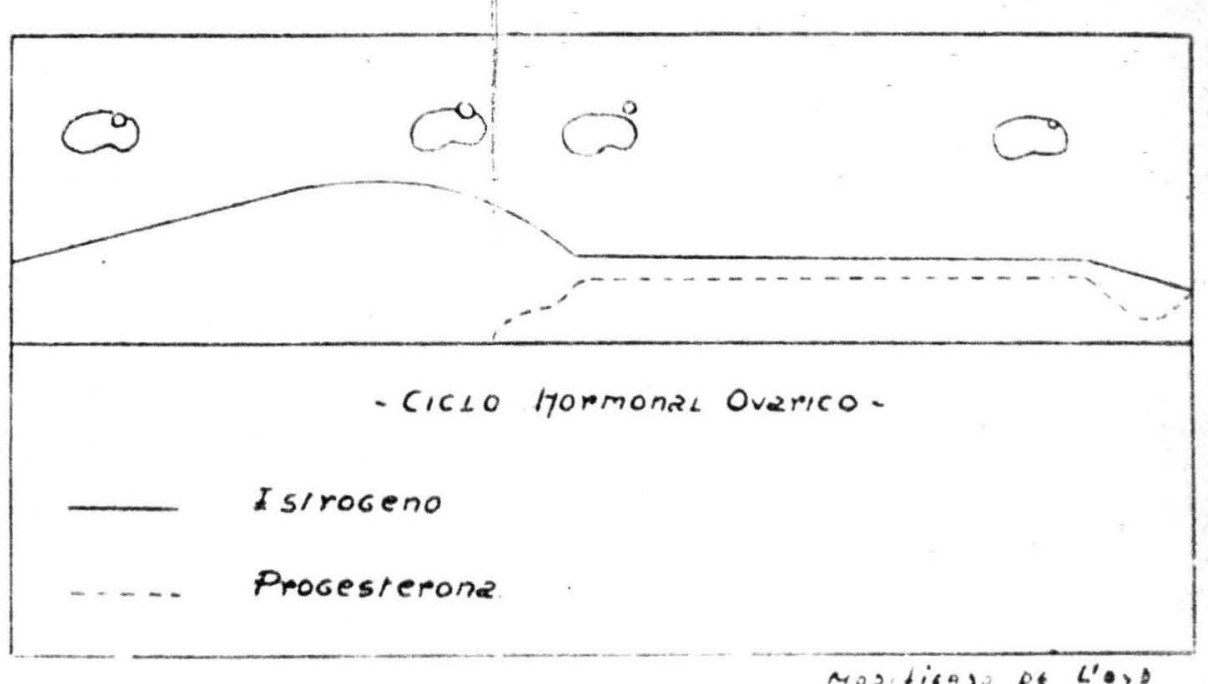

(GRAFICA NO 3)

Estrogenos y progesterona no son antagónicos sino sinergicos. Sus fuentes de producción no están únicamente en el ovario; los hay en otras glándulas.

El testículo produce en sus canales seminiferos la célula germinal masculi na o espermatozoide. El estímulo de este epitelio glandular, está dado por FSH. Las células intersticiales o de Leydig, bajo la acción de LH, produce los andró genos cuya fuente está también en la corteza adrenal.

La testosterona es el principio activo de la célula intersticial obtenido en forma cristalina y la androsterona fue sintetizada a partir del colesterol y se en cuentra en la orina de machos y hembras ( ${ }^{\bullet}$ ). El mecanismo hipofiso-testiculat es semejante al hipofiso-ovárico y son valederos los conceptos antes expueston. de inhibición de la glándula blanco sobre la central. Dosis altas o continuas de testosterona pueden producir una azoospermia temporal o permanente por inhi 
bición de FSH; este fenómeno también se observa con administración de estrógenos.

Como se dijo arriba los andrógenos tienen bajo su dominio el desarrollo y sostenimiento de los caracteres sexuales primarios y secundarios, interviniendo en el desarrollo del pene, testiculos, canales deferentes, vesículas seminales, prós tata, etc., asi como en lia distribucion del vello y grasa, caracteristicas esquele ticas, voz y hábito.

La hormona Foliculocstimulante es dosificable en el humano y su estudio a lundamental en los síndromes primitivamente hipofisarios o gonadales. Los andrógenos se excretan en forma de 17 cetoesteroides y la diferencia de cifras entre los dos sexos, representa la fracción testicular. Los estrógenos pueden dosificarse en orina o sangre y como ya se dijo, el pregnandiol urinario representa el nivel de progesterona en la sangre.

\section{Patologín}

Los sindromes en Endocrinologia se clasifican en hiper e hipofuncionantes. Consideramos lo que se refiere a hipófisis, testículo y ovario.

Hipófisis: Hipofunción ( $\left.{ }^{10}\right)$. Los síndromes varían de acuerdo con la edad del paciente y comienzo de la enfermedad.

Simmonds: generalmente debido a un tumor no funcionante de tipo destructivo o de vecindad. Otras veces por procesos crónicos de fibrosis o infección dentro de los cuales cabrían la sífilis y TBC. Resulta cuando se encuentra una interferencia severa de la secreción en cualquier edad. Puede clasificarse dentro de este síndrome el Mixedema pituitario en que hay hipotiroidismo secundario sin elevación de colesterol y con una baja sexual notable. Shechan en 1937 describió un síndrome que identificó a las necrosis que siguen al parto con hemo rragia y shock y so la llama enfermedad de Sheehen. No siempre en él se en cuentra caquejia y cuando la hay es mucho menos severa que en el Simmonds puro. También puede haber un Addison hipopituitario en que es de valor el uso de ACTH. Clínicamente, estos síndromes se caracterizan por una baja uni versal de las funciones de la hipofisis anterior.

Cuando predomina la hipofunción de somatotrofina y gonadotrofina se obtienen los infantilismos y enanismos tipo Levi Lorain cuyas causas más frecuentes son tumores, infartos de los vasos de la glándula, infección o simplemente una falta de desarrollo de la misma. El déficit hipofisario no es nunca tan severo como en el Simmonds y clinicamente son pacientes armónicamente enanos $\mathrm{e}$ in maduros sexuales. El síndrome de Froehlich o adiposo genital se debe a un tumor de la hipófisis o del hipotálamo, con obesidad e hiposexualismo. Es raro el ver dadero y muy escaso en el adulto. Deben tenerse en cuenta la existencia de ver daderos signos hipotalámicos para su diagnóstico positivo.

Hiperfunción acromegalia y gigantismo cuando hay hiperfunción de la hormona del crecimiento. La lesión esencial es un adenoma eosinófilo. El gigantismo resulta cuando la lesión se hace sobre un individuo joven con sus epifisis abiertas. 
Cuando va están cerradas, el sobre-estímulo de hormona produce los cambins esqueléticos tupicos de la acromegalia. Se han descrito una serie de tumores on intervencien directa sobre la secreción de gonadotrofinas (").

la cnfermedad de Cushing puede también clasificarse dentro de los sin dromes hiperfuncionantes del eje hipofiso-suprarrenal on consecuencior gona dakes claras como amenorrea $y$ anosulacion

Orano: Fuera de la maduracion del ovelos de la preparacion homonal

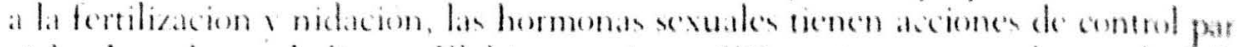

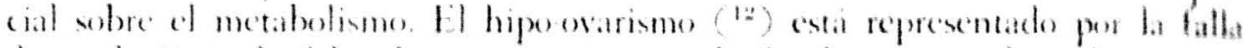
de ovulacion y la falta de preparacion para la fertilizacion y el implante mu cho hipoovarismo o hallas ovaricas sucken manifestarse en varias formas de desordenes monstruales. La sangria uterina no es funcion fundamental del ovario y no siempre hay ovulacion en una mujer cumenorreica. Hipo-ovarismos con aumento de $F S H$; Eunucoidismo no siempre es de diagnóstico facil. So caracteriza por menarquia retardada despues de los 17 anos, asociada a neutra. lidad sexual, alargamiento del esqueleto, cifras altas de FSH, ausencia de ma. rifestaciones estrogenicas; el desarrollo de los organos sexuales es precario. Sindrome ménopansico: se caracteriza por la sesación permanente de las mens truaciones; comúnmente se $k$ confunde con el sindrome de castración $y$ di matterium. La edad del paciente y estado funcional del ovario influyen en la severidad de los sintomas en el sindrome de castración; por debajo de los 25 opor encima de los 55 años, la castracion raramente produce un disturbio sero. Los sintomas de envejecimiento del ovario se caracterizan por cambios vasomotores, desarreglos menstruales y atrolia genital. El climaterio se caracteriza por cambios emocionales, laxitud, parestesias, dolores osteoarticulares desarre glos scexuales y obesidad. No pocas veces los dos sindromes se sobreponen peto esto no es suficiente para que se confundan. Sindrome de Turner: caracterizado por: agenesia del ovario, hipoplasia genital, amenorrea primaria y bija estatura, pero no de tipo enanismo; cubitus valgus y cuedlo palmado, algunas veces desat denes oculares y pueden exiseir tambien malformaciones congénitas pelimor fas. Es fundamental el hallazgo de valores altos de FSll para de diannósere (13).

Sindrome de hipotancion ovárica con valores normales de FSH: netro pata hemorragica o hemorragia uterina funcional (14). Ia causa basica es una disfuncion hormonal y como causas advuvantes podrian invocarse factores nu tricionales o psicógenos y deficiencias vitaminicas. Amenorrea hipotalamica $\left({ }^{5}\right)$, Es de frecuente observacion el hallazge de alteraciones de ciclo menstrual debi das a un trastorne funcional del sistema nervioso central; especialmente fie cuente en pacientes con lesiones mentales y con mayor incidencia en las do lipo depresiso. Tampoco es raro ver la interrupción del ritmo menstrual por cambios ambientaks temor o angustla. Al examen genital se encuentra atrofia de la mucose vaginal y del endometrio. Es fundamental en el diagnostico de esta clase de amenorrea el trauma psiquico, antes del comienze de la enterme dad. EI FSH es normal y no se puede demostrar un eleco estrogenico en lon 
cejides pelvicos. Dentro de los sindromes sin variacion de FSt cabria el de Stein con anovulacion, amenorreat e hirsutismo.

sindromes hiperfancionantes cestan representades por tumores del ovario.

Tumores feminizentes a de la gramulos an mediano hiperestrinismo.

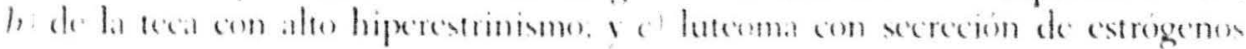
1 pogesteromes.

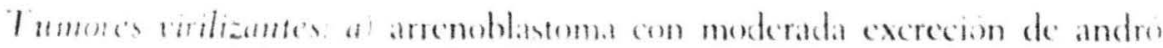

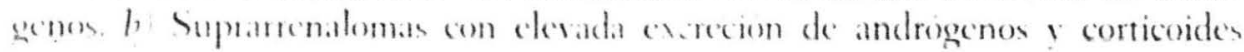

Feminizante a no el coriocarcinomat con alta excecion de FSH y modera da de estrogenos. Se citaran el disgeminoma y el estruma ovari.

Sindrome de hipofuncion testicular con alta FSH.

Eunucoidismo: caracterizado por neutralidad sexual y alargamiento del es queleto, azoospermia, altos valores de FSH; desarrollo precario de los organos sevuales.

Sindrome de Klinefelter: caracterizado por hipotrofia testicular, azoospermia. ciracteres sexuales relativamente normales, cifras muy altas de FSH en la orina $\checkmark$ algunas veces ginecomastia. No es raro encontrar valores subnormales en la sicrecion de 17 cetoesteroides.

Tumores hormomalmente activos del testiculo (1"i). Casi siempre de origen canceroso y de patologia compleja: a de las celulas de Levdig con excrecion de andrigenos, en los ninos pueden producir una pubertad percoz; b) Células de Sertoli: cxcretan estrogenos y son feminizantes; y cl cl coriocpitelioma. Es de valor la sosilicación de FSII y gonadotrofina corionica en estos pacientes. No hay que olvidar yue el 95\% de Fon tumores del testiculo son malignos y que l:e incidencia de malignidad es muy grande cuando el testículo está ectopico ( ${ }^{7}$ ?

\section{B I B LIOG R A F I A}

1.-Houssy: "Fisiologia de la Hipofisis". Tratado de Endocrinologia Clínica. Moussio Fournier. Pág. 258 y sig. Kraft. Tomo I. 1950. Buenos Aires.

2.-Houssay. "Fisiologia Humana". Pág. 806. "El Ateneo". Buenos Aires. 1950. Book I. Abstracts of Lectures, Fifth annual postgraduate Assembly. By Endocrine Society, 1952

3.-Klinefelter: "Chemistry and Physiology of the anterior Pituitary". Pag, 31.

4.-Nierburgs: "Hormones in clinical practice". Pág. 18 y sig. Hoeber. Londres, 1950

5 - Lloya: "Chemistry and Physiology of ovaries; Physiology of menstruation". Book 4, Pag. 1. Abstracts of lectures, Fifth annual postgraduate Assembly. 1952. By Endocrine Society.

1i - Verós A.: "Conferencia sobre estrogenos". Clínica Camero. Inédito. 1952. Bogotá.

7 - Hamilton J. B.: "El vello axilar como indice cuantitativo del estado endocri. no". Progresos de la endocrinología clínica. Soskin. Pag. 548. Científico Médica. 1951. Madrid. 
8.-Nierburgs: "Hormones in clinical practice". Pag. 169. Hoeber. Londres. 1950.

9.-Nierburgs: "Hormones in clinical practice". Pag. 180. Hoeber. Londres. 1950.

10.-Escamilla: "Anterior Pituitary Hipofunction Syndromes: Panhipopituitarism Simmonds cachexia, Sheehan's Syndrome, Anorexia nervosa and Frohlich's syndrome". Book II, Pag. 38. Abstract of lectures, Fifth annual postgraduate Assembly. By Endocrine Society. 1952.

11. -Klinefelter: "Other Anterior Pituitary Syndromes". Book II, Pag. 57. Abstracts of lectures, Fifth annual postgraduate Assembly. By Endocrine Sociey. 1952.

12.-Brown: "Ovarian Hipofunction Syndromes: a) Hipergonadotropic Syndromes castrates; Menopause; Hipogonadism". Book IV, Pag. 6. Fifth annusl postgra. duate Assembly. By Endocrine Society. 1952.

13. - Turner: "Ovarian Hipofunction Syndromes: Hipergonadotropic Syndrone: Ovarian agenesis and Turner's Syndrome". Book IV, Pag. 11. Fifth annual fost-gra. duate Assembly. By Endocrine Society. 1952.

14.-Greenblatt: "Ovarian Hipofunction Syndromes: c) Normogonadotroplc Syn. dromes: Metropathia Hemorrhagica: Dysmenorrhea: Endometriosis". Book IV. Pag. 24. Fifth annual postgraduate Assembly. By Endocrine Society. 1982.

15. -Reifenstein: "Ovarian Hipofunction Syndromes: Normogonadotropic Syndromes: Hypothalamic Amenorrea". Book IV, Pag. 33. Fifth annual postgraduate Assembly. 1952.

16. -Twombly: "Tumores hormonalmente activos del testículo". Pág. 523. Soskin. Progresos de la Endocrinologia Clínica. Científico-médica. 1951. Madrid.

17. - Simmonds: "Testicular Hiperfunction Syndromes and Tumors". Leydig Celt, Chorionepithelioma, and others. Book IV, Pag. 94. Fifth annual postgradunte Assembly. By Endocrine Society. 1952. 